\title{
Diacronie
}

Studi di Storia Contemporanea

$\mathrm{N}^{\circ} 34,2 \mid 2018$

Scuola e società in Italia e Spagna tra Ottocento e Novecento

\section{Alessio Gagliardi, Il 77 tra storia e memoria}

Leonardo Recupero

\section{(2) OpenEdition \\ Journals}

\section{Edizione digitale}

URL: http://journals.openedition.org/diacronie/7987

DOI: $10.4000 /$ diacronie. 7987

ISSN: 2038-0925

Editore

Association culturelle Diacronie

Notizia bibliografica digitale

Leonardo Recupero, «Alessio Gagliardi, // 77 tra storia e memoria », Diacronie [Online], №34, 2 | 2018, documento 17, Messo online il 29 juin 2018, consultato il 23 septembre 2020. URL : http://

journals.openedition.org/diacronie/7987 ; DOI : https://doi.org/10.4000/diacronie.7987 


\title{
Diacronie
}

Studi di Storia Contemporanea

34, $2 / 2018$

Scuola e società in Italia e Spagna tra Ottocento e Novecento

\section{RECENSIONE: Alessio GAGLIARDI, Il 77 tra storia e memoria, Roma, manifestolibri, 2017, 128 pp.}

\author{
A cura di Leonardo RECUPERO
}

Per citare questo articolo:

RECUPERO, Leonardo, «RECENSIONE: Alessio GAGLIARDI, Il 77 tra storia e memoria, Roma, manifestolibri, 2017, 128 pp.», Diacronie. Studi di Storia Contemporanea : Scuola e società in Italia e Spagna tra Ottocento e Novecento, 34, 2/2018, 29/06/2018,

URL: < http://www.studistorici.com/2016/12/29/recupero_numero_34/ >

Diacronie Studi di Storia Contemporanea $\rightarrow$ http://www.diacronie.it Rivista storica online. Uscita trimestrale.

redazione.diacronie@hotmail.it

Comitato di direzione: Naor Ben-Yehoyada - João Fábio Bertonha - Christopher Denis-Delacour - Maximiliano Fuentes Codera Anders Granås Kjøstvedt - John Paul Newman - Deborah Paci - Niccolò Pianciola - Spyridon Ploumidis - Wilko Graf Von Hardenberg

Comitato di redazione: Jacopo Bassi - Luca Bufarale - Gianluca Canè - Luca G. Manenti - Fausto Pietrancosta - Alessandro Salvador - Matteo Tomasoni - Luca Zuccolo 


\title{
17/ RECENSIONE: Alessio GAGLIARDI, Il 77 tra storia e memoria, Roma, manifestolibri, 2017, 128 pp.
}

\author{
A cura di Leonardo RECUPERO
}

Il decennio degli anni Settanta fu un periodo di profonde trasformazioni per l'Italia. Esse investirono le strutture economiche, politiche, sociali e, più in generale, culturali del paese. L'Italia, pienamente inserita nelle dinamiche bipolari della guerra fredda, soffrì della crisi economica e finanziaria che investì pesantemente tutto il mondo occidentale in quel decennio ${ }^{1}$. Aggravò la situazione il fatto che, nel nostro paese, la crisi economica coincise con la crisi del sistema politico nella formula del centro-sinistra. Il decennio vide il susseguirsi di governi instabili e con maggioranze improvvisate: ciò «impedì che venissero assunti provvedimenti efficaci in campo monetario e creditizio, che sarebbero stati necessari per contrastare gli agenti propulsivi dell'inflazione $»^{2}$. Contemporaneamente il PCI allargò la sua base elettorale e intravide l'incarico di governo a portata di mano, dopo un intero trentennio di relegazione all'opposizione e di egemonia della DC. Furono gli anni del «compromesso storico» e del "governo della non sfiducia» (1976-78). A differenza degli altri stati europei, in Italia il boom economico non vide il necessario completamento del welfare lasciando così il mondo del lavoro nell'incompletezza dei diritti sociali; ciò, non appena si affacciò la crisi, generò un reale impoverimento delle famiglie e innescò la crescita della conflittualità sociale. Lo stesso PCI non seppe formulare un patto fiscale tra Stato e cittadini a tutela dei servizi che il primo era chiamato a garantire3. La logica dei comunisti e del loro sindacato rimase incastrata nella difesa di un modello di mercato del lavoro che cambiò velocemente: la classe - o forse, meglio, la generazione - operaia che si garantì i diritti con le lotte iniziate nel 1969 non era più quella emarginata socialmente; al suo posto emersero ampi strati di povertà e di disoccupazione (spesso giovanile) che non erano coperti dai diritti

\footnotetext{
${ }^{1}$ Cfr. ROMITELLI, Valerio, Introduzione, in DE BERNARDI, Alberto, ROMITELLI, Valerio, CRETELLA, Chiara (a cura di), Gli anni Settanta. Tra crisi mondiale e movimenti collettivi, Bologna, Archetipolibri, 2009, pp. VII-XXI, p. $\mathrm{X}$.

${ }^{2}$ DE BERNARDI, Alberto, I movimenti di protesta e la lunga depressione dell'economia italiana, in ibidem, pp. 119135, p. 123.

${ }^{3}$ Cfr. ibidem, pp. 123, 126.
} 
sindacali e che si trovavano di fronte, per l'appunto, ad un nuovo modello di mercato del lavoro più precario, meno garantito e non più fondato sulla stabilità della grande impresa 4 .

Fu in questo contesto che si accese la stagione di contestazioni del 1977, spesso studiata come epigono del '68. Ma

pur essendoci un filo rosso che unì i due cicli di contestazione, il Settantasette fu altro rispetto al Sessantotto. Fu una sorta di figlio adolescente - più impertinente, più impaziente e più disincantato - che recuperò le premesse della generazione precedente portandone all'estremo gli esiti, tanto da stravolgere e talvolta negare quelle stesse premesse 5 .

È possibile studiare questo ciclo di contestazioni da molteplici punti di vista, così come altrettanto vari furono gli eventi (e la loro portata) che caratterizzarono quell'anno. Furono molti gli attori protagonisti degli eventi, che spaziarono dagli studenti universitari ai giovani lavoratori emarginati; furono molti i temi di contestazione: dal contesto politico, economico, sociale di quegli anni fino ai dibattiti sui principi teorici che muovevano le più diverse frange di contestatori (che, è bene ricordarlo, furono tutti da ascrivere alle diverse anime che componevano la sinistra italiana di quegli anni). Furono molti i luoghi in cui il movimento si mosse e manifestò la sua carica eversiva, sempre però in contesti metropolitani: dal nord e dal centro, dove le proteste furono più accese e sicuramente più visibili da un punto di vista mediatico, al sud, "ampiamente sincronizzato col resto d'Italia». Furono molti i mezzi che vennero utilizzati per esprimersi: dalle manifestazioni in piazza ai cortei, dai giornali e dai fogli politicizzati alle nuove radio libere e sovversive, dalle scritte sui muri agli slogan di piazza, dai convegni nazionali, come quello a Bologna di settembre, alle grosse contestazioni universitarie, come quella a Roma di febbraio dove il sindacalista della Cgil Luciano Lama fu cacciato dalla Sapienza. E ancora: dai nuovi linguaggi ironici che soppiantavano e schernivano la tradizione politica di sinistra (ben incarnata dal segretario del PCI Enrico Berlinguer) e i suoi modi seri e megalitici, ai nuovi modi di concepire e mettere in atto la politica; dalle feste creative piene di balli, colori, droghe, erotismo e coinvolgimento emotivo, alla violenza di piazza e di corteo, alle risse, incendi, espropri proletari, alle vere e proprie guerriglie cittadine, ai feriti e ai morti del marzo bolognese, dell'aprile e del maggio romani, del maggio milanese e dell'ottobre torinese.

\footnotetext{
${ }^{4} \mathrm{Cfr}$. ibidem, pp. 132-133. Sulla crisi economica e politica italiana si consultino anche i primi due capitoli di FALCIOLA, Luca, Il movimento del 1977 in Italia, Roma, Carocci, 2015.

${ }^{5}$ FALCIOLA, Luca, op. cit., pp. 256-257. Il dibattito storiografico sui rapporti tra '68 e '77 costituisce una questione complessa non affrontabile in questa sede, si rimanda dunque a: DE BERNARDI, Alberto, ROMITELLI, Valerio, CRETELLA, Chiara (a cura di), op. cit., passim; GAGLIARDI, Alessio, Il 77 tra storia e memoria, Roma, manifestolibri, 2017.

${ }^{6}$ GAGLIARDI, Alessio, op. cit., p. 23.
} 
Il discorso sulla violenza assume rilevanza fondamentale nella discussione storica del Settantasette, poiché influenzato enormemente dall'opinione che si impresse nell'immaginario collettivo della società italiana a partire da quei cupi «anni di piombo». Certo è che se da un lato il racconto del movimento fu egemonizzato dalla sua radicalizzazione e militarizzazione che coincise con il racconto mass-mediatico, dall'altro esiste anche una memoria pacificata, spesso da parte di ex militanti7. Bisogna poi distinguere tra i diversi tipi di violenza e i diversi soggetti che la praticarono, tra la sua effettiva messa in atto e la sua legittimazione ideologica espressa per vie mediatiche. Bisogna distinguere tra la violenza compiuta dai militanti del movimento e quella repressiva, spesso sottaciuta, delle forze dell'ordine e degli apparati dello Stato ${ }^{8}$.

Alessio Gagliardi ${ }^{9}$ si inserisce nel dibattito sul Settantasette con una prospettiva che non vuole essere né una ricostruzione cronologica di quell'anno, né una interpretazione tematica che voglia ripercorrerlo alla luce di un esplicito filone narrativo, né tantomeno si prefigge di mettere a punto una mera rassegna storiografica ${ }^{10}$. Il suo scopo emerge sin dall'Introduzione: proporre un primo bilancio critico degli studi per sviluppare una riflessione che dia conto della rilevanza storica del Settantasette nel più ampio orizzonte della storia dell'Italia repubblicana ${ }^{11}$, nel quale tutt'oggi fatica ad inserirsi. L'aspirazione a ricondurre tale evento a processi e trasformazioni di medio e lungo periodo è infatti una costante del volume. La si ritrova declinata variamente fra gli otto capitoli che Gagliardi dedica a ciascun tema caratterizzante il Settantasette, e viene discussa in virtù di un esteso apparato di ricerche sia storiografiche che memorialistiche: rinnovare la riflessione sul Settantasette, da un lato attraverso ciò che di esso è a tutti gli effetti entrato nella storia e dall'altro tenendo sempre presente il vissuto dei militanti che ne tramandarono la memoria ${ }^{12}$, spiega anche la scelta del titolo del volume.

Il primo capitolo si concentra sulla percezione generale che si ebbe al farsi presente dello «strano movimento» caratterizzato sin da subito da profonde contraddizioni; la conclusione che se ne trae è quella di una nuova emergente concezione di politica, strana perché costitutivamente

\footnotetext{
${ }^{7}$ Cfr. ibidem, p. 34.

${ }^{8}$ Il dibattito storiografico sulla violenza del '77 (e, più in generale, sulla violenza politica negli anni Settanta) è ampio e, anch'esso, non affrontabile in questa sede. Si rimanda dunque a: ibidem, pp. 33-41 (secondo capitolo); FALCIOLA, Luca, op. cit., pp. 196-253 (ultimi due capitoli); BELLASSAI, Sandro, Un trauma che si chiama desiderio. Per una storia del Settantasette a Bologna, in DE BERNARDI, Alberto, ROMITELLI, Valerio, CRETELLA, Chiara (a cura di), op. cit., pp. 213-234; per un approccio che guardi al caso italiano inserito nel contesto internazionale della pratica della violenza politica si veda TOLOMELLI, Marica, Militanza e violenza politicamente motivata negli anni Settanta, in ibidem, pp. 192-210.

${ }^{9}$ È ricercatore all'Università di Bologna, dove insegna Storia contemporanea. I suoi temi di ricerca vertono sul fascismo, sulle istituzioni economiche e le organizzazioni sindacali nell'Italia contemporanea, le culture politiche dell'antifascismo, il pensiero politico di Antonio Gramsci, i processi di internazionalizzazione in campo economico e istituzionale tra Otto e Novecento, e sui movimenti sociali degli anni Sessanta e Settanta.

${ }^{10} \mathrm{Cfr}$. GAGLIARDI, Alessio, op. cit., p. 13.

${ }^{11} \mathrm{Cfr}$. ibidem.

${ }^{12} \mathrm{Cfr}$. ibidem, p. 82.
} 
poco inquadrabile con gli strumenti tradizionali della storia della politica. Quest'ultima stava infatti attraversando i profondi «processi di trasformazione [che] erano in atto, e [di cui] il '77 [...] portò fortemente impresso il segno» ${ }^{13}$. I due capitoli seguenti si soffermano sul tema cardine della violenza che da un lato caratterizzò il movimento dall'interno (violenza praticata) e dall'altro lo circondò dall'esterno, come la violenza subita nella repressione e quella terroristica della lotta armata di cui il movimento fu accusato senza i dovuti distinguo. Quello relativo ai diversi modi e alle diverse forme di violenza è infatti

uno sforzo di distinzione a cui non si può rinunciare, e che necessita che il discorso sulla violenza politica diventi parte integrante di un più ampio discorso sulla storia politica, sociale e culturale dell'evento indagato. [...] solo ricostruendo la complessità dei contesti e, nel contempo, culture politiche, obiettivi e percezioni dei protagonisti, si può sperare di evitare equivoci e visioni parziali. È un lavoro [...] di cui la comprensione del '77, negli aspetti più complessi e nascosti, può sicuramente giovarsi ${ }^{14}$.

Il quarto capitolo pone l'accento sull'ala creativa del movimento e sulla sua capacità di mostrare una potenza eversiva innovativa anche rispetto alle manifestazioni di violenza. I due estremi però non vanno immaginati come due realtà diverse e parallele ognuna con il proprio raggio di influenza sulla mobilitazione, bensì come due facce di una stessa medaglia, due aspetti che convissero e si attraversarono: chi si esprimeva creativamente e festosamente spesso aderiva anche all'eversione di tipo violento, o ancora sintetizzava i due atteggiamenti nelle taglienti armi dell'ironia, ben diversa dalla felicità spensierata ${ }^{15}$. Anche su questo fronte, poi, si può affermare che le

pratiche creative del '77 si inserivano in un processo, già ben avviato, di profondo rinnovamento della informazione «ufficiale», o almeno di suoi vasti settori, all'insegna di un minore ossequio verso i detentori del potere politico e un maggiore coinvolgimento dei cittadini. Guardando il panorama mediatico il decennio appare [...] un periodo di grande ricchezza e creatività, con pochi eguali nella storia del Novecento italiano ${ }^{16}$.

Dopo aver delineato un affresco in grado di restituire la multilateralità del movimento del '77, negli ultimi capitoli Gagliardi fornisce una chiave di lettura più incentrata sulla rilevanza storicopolitica di quell'evento e che ne costituisce una delle più importanti eredità: la rottura con la tradizione politica della sinistra "storica" verso una radicale rimodulazione delle sue culture

\footnotetext{
${ }^{13}$ Ibidem, p. 31.

${ }^{14}$ Ibidem, p. 39.

${ }^{15}$ Cfr. ibidem, pp. 63-64.

${ }^{16}$ Ibidem, p. 62.
} 
politiche. Difatti, nonostante si consideri spesso quell'anno come l'epilogo della stagione movimentista inaugurata nel '68,

dopo il '77 in Italia l'impegno collettivo così come i cicli della protesta non evaporarono, nonostante il prevalere di sentimenti conservatori e neoliberali. Sottotraccia, e in forme meno dirompenti, avrebbero continuato anche negli anni e decenni successivi a segnare con la propria presenza il panorama politico e sociale nazionale. [...] Lungo questa linea si sarebbero riorganizzate le principali culture politiche ma anche le mobilitazioni collettive e le proteste extrapartitiche, benché fortemente ridimensionate ${ }^{17}$.

Le trasformazioni in corso nel mondo della sinistra italiana sono da collocarsi nella storia più ampia della sinistra tradizionale europea, che perseverò in una sempre più anacronistica visione della società e dei suoi conflitti in termini di classe ${ }^{18}$. È da leggere in questa stessa prospettiva di solidarietà con le tendenze europee l'atteggiamento antagonista del PCI e della Cgil nei confronti del movimento. Solo poche voci, critiche ed insofferenti quanto inefficaci, si levarono dalle frange del Partito comunista verso le ortodossie radicate dei propri gruppi dirigenti, resi ancor più rigidi dalla pressione della contestazione. Il movimento, dunque, riuscì ad influenzare le istituzioni della sinistra tradizionale solo in minima parte, non riuscendone a sfondare la «granitica linea ufficiale» ${ }^{19}$.

Propongo in sede conclusiva una breve e personale riflessione sull'operazione storiografica che compie Gagliardi. Appare animata dalla impellente necessità di riflettere sugli anni Settanta, e in particolare su un evento-anno che, con la sua ricchezza esplosiva, seppe riassumere ed esprimere le profonde tensioni che attraversarono un decennio tumultuoso e decisivo della storia del Novecento. La ricostruzione di Gagliardi esprime dunque l'esigenza di tornare a riflettere sul '77, mostrandoci la sua crucialità per la comprensione dell'oggi. Ciò lo pone in evidente continuità con la storiografia che costituisce la sua base di ricerca, e in particolare con lo studio di Falciola, «la prima ricerca sistematica basata su un ricco apparato di fonti» ${ }^{20}$. Infine, consapevole dei rischi che comporta un approccio storiografico al Settantasette, l'autore sembra seguire un'indicazione di metodo suggerita in maniera efficace da Sandro Bellassai: «né celebrare [...], né condannare, ma davvero ripensare gli anni Settanta» ${ }^{21}$.

\footnotetext{
${ }^{17}$ Ibidem, p. 73-74.

${ }^{18}$ Cfr. ibidem, p. 77.

${ }^{19}$ Ibidem, p. 94-95.

${ }^{20}$ Ibidem, p. 10.

${ }^{21}$ BELLASSAI, Sandro, op. cit., p. 234.
} 


\section{L'AUTORE}

Leonardo RECUPERO è iscritto al terzo anno del corso di laurea in Filosofia dell'Università di Bologna. Attualmente svolge un tirocinio curriculare presso la rivista «Diacronie. Studi di storia contemporanea». I suoi interessi principali e l'orientamento dei suoi studi vertono principalmente sulla storia, nonché sulla storia del pensiero dell'età moderna e contemporanea.

URL: < http://www.studistorici.com/progett/autori/\#Recupero > 